\title{
Primer registro del flebotomíneo Warileya (Hertigia) hertigi en Antioquia, Colombia
}

\author{
Eduar Elías Bejarano', Rafael José Vivero², Sandra Uribe ${ }^{3}$ \\ Investigaciones Biomédicas, Universidad de Sucre, Sincelejo, Colombia \\ 2 Programa de Estudio y Control de Enfermedades Tropicales, Universidad de Antioquia, Medellín, Colombia \\ 3 Grupo de Investigación en Sistemática Molecular, Universidad Nacional de Colombia, Medellín, Colombia
}

Introducción. El género Warileya es uno de los taxones menos conocidos de los flebotomíneos, el cual está integrado por solo nueve especies: Warileya (Warileya) phlebotomanica, W. (Hertigia) hertigi, $W$. (W.) rotundipennis, $W$. (W.) nigrosacculus, $W$. (W.) yungasi, $W$. (W.) fourgassiensis, W. (W.) lumbrerasi, W. (W.) euniceae y W. (W.) leponti.

Objetivo. Registrar el hallazgo de una especie del género Warileya en Antioquia, Colombia.

Materiales y métodos. Los flebotomíneos se recolectaron en una caverna de la Reserva Natural Cañón del Río Claro del municipio de San Francisco, departamento de Antioquia, Colombia. El muestreo entomológico se hizo con una trampa de luz de tipo CDC, durante tres noches consecutivas, en mayo de 2008. La determinación taxonómica se basó en la revisión del material tipo de la especie y de las claves estándar para flebotomíneos americanos.

Resultados. Se identificaron taxonómicamente cinco machos y dos hembras de flebotomíneos como W. $(H$.) hertigi. En ambos sexos fue notoria la ausencia de cicatrices de setas en el anepisternón, el proepimerón y el clípeo, la presencia de dos hileras transversales de cicatrices de setas en los tergitos, y la longitud corta de la vena gamma.

Conclusión. El hallazgo de $W$. (H.) hertigi incrementa a 64 el número de especies de flebotomíneos encontradas en el departamento de Antioquia. En total, 164 especies de flebotomíneos han sido registradas en Colombia.

Palabras clave: Psychodidae, leishmaniasis, Colombia.

doi: https://doi.org/10.7705/biomedica.v38i0.3625

First record of the sand fly Warileya (Hertigia) hertigi from Antioquia, Colombia

Introduction: The genus Warileya is one of the least-known taxa of sandflies, comprising only nine species, i.e., W. (Warileya) phlebotomanica, W. (Hertigia) hertigi, W. (W.) rotundipennis, W. (W.) nigrosacculus, W. (W.) yungasi, W. (W.) fourgassiensis, W. (W.) lumbrerasi, W. (W.) euniceae and $W$. (W.) leponti.

Objective: To document the presence of a species of the genus Warileya in Antioquia, Colombia.

Materials and methods: Sandflies were collected in a cavern of the Cañón del Río Claro Natural Reserve, of the municipality of San Francisco, Antioquia department, Colombia. Phlebotomine sampling was carried out using a CDC light trap during three consecutive nights in May of 2008. Taxonomical determination was based on a revision of the type material of the species and through the use of standard keys for American sandflies.

Results: Five male and two female sandflies were taxonomically identified as $W$. (H.) hertigi. In both sexes, the absence of setal scars in the anepisternum, proepimeron and clypeus; the presence of two transverse rows of setal scars in the tergites; and the short length of the vena gamma were notable.

Conclusion: The finding of $W$. $(H$.) hertigi increases the number of sandfly species found in Antioquia department to 64 . In total, 164 sandfly species have been recorded in Colombia.

Key words: Psychodidae, leishmaniasis, Colombia.

doi: https://doi.org/10.7705/biomedica.v38i0.3625

\footnotetext{
Contribución de los autores:

Eduar Elías Bejarano: determinación taxonómica de la especie

Rafael José Vivero: recolección de flebotomíneos, montaje entomológico y determinación taxonómica

Sandra Uribe: diseño del estudio

Todos los autores participaron en la escritura del manuscrito.
} 
Los flebotomíneos son ampliamente reconocidos como vectores de microorganismos patógenos, entre los cuales sobresalen los protozoos del género Leishmania Ross, 1903, que se alojan en la piel, las mucosas o las vísceras de los mamíferos (1). La mayoría de los flebotomíneos descritos en el Nuevo Mundo pertenecen al género Lutzomyia França, 1924, que reúne más de 500 especies a la fecha, seguido por Brumptomyia França y Parrot, 1921, con 26 especies, y Warileya, Hertig, 1948, con solo nueve especies (2).

A diferencia de las especies de Lutzomyia y Brumptomyia, que presentan una amplia distribución en el neotrópico, las especies del género Warileya se concentran en siete países: Costa Rica, Panamá, Colombia, Perú, Ecuador, Bolivia y Guayana Francesa (3). Aunque las especies de Warileya no se habían asociado con la transmisión de agentes patógenos al humano, recientemente $W$. (Warileya) rotundipennis Fairchild y Hertig, 1951, se encontró infectada con parásitos del subgénero Leishmania (Viannia) en un foco de leishmaniasis cutánea de Colombia (4).

Durante un estudio entomológico desarrollado en el departamento de Antioquia, se recolectaron flebotomíneos que correspondían a una especie de Warileya no registrada en esta región, los cuales se reportaron inicialmente como Warileya spp. (5) hasta tanto se pudiera acceder al tipo de la nomenclatura para corroborar su identidad. La comparación de estos ejemplares con el tipo indica que pertenecen a la especie $W$. (Hertigia) hertigi (Fairchild, 1949), la cual es registrada por primera vez en Antioquia, Colombia, en la presente comunicación breve.

\section{Materiales y métodos}

Los flebotomíneos se recolectaron en la Reserva Natural Cañón del Río Claro (553'15.30”N, 74'51'18.65"O; WGS 84), vereda Altavista, del municipio de San Francisco, departamento de Antioquia, Colombia. Esta reserva se encuentra ubicada a $324 \mathrm{msnm}$, en la estribación suroriental de la Cordillera Central de Los Andes. Ecológicamente, la zona corresponde a bosque húmedo

Correspondencia:

Eduar E. Bejarano, Universidad de Sucre, Carrera 14 NN$^{\circ} 16 \mathrm{~B}-32$, Sincelejo, Colombia

Teléfono: (575) 282 0830; fax: (575) 2821240

eduarelias@yahoo.com

Recibido: 11/10/16; aceptado: 30/07/17 tropical, con registros de temperatura media anual de $26{ }^{\circ} \mathrm{C}$, aproximadamente, y humedad relativa de 80 a $85 \%$.

Para la recolección de los flebotomíneos, se usó una trampa de luz de tipo CDC, que se instaló a 1,5 $m$ del suelo, dentro de una caverna, a un lado del sendero ecológico paralelo al río Claro. La trampa se accionó entre las 18:00 y las 06:00, durante tres noches consecutivas, en mayo de 2008.

Los ejemplares fueron aclarados en lactofenol (ácido láctico y fenol, en proporción 1:1) durante 24 horas y montados en láminas portaobjeto usando bálsamo de Canadá.

Las estimaciones morfométricas de cada ejemplar se hicieron con un micrómetro de ocular en un microscopio Carl Zeiss Primo StarTM. El material entomológico está depositado en la "Colección de Vectores y Hospedadores Intermediarios de Enfermedades Tropicales (VHET)" del Programa de Estudio y Control de Enfermedades Tropicales (PECET) de la Universidad de Antioquia en Medellín, Colombia.

La identificación taxonómica de los flebotomíneos se basó en las claves de Young y Duncan (6), y de Galati (3), y en los artículos con las descripciones originales de las nueve especies de Warileya hasta ahora conocidas (7-16). Además, se examinó el material tipo de $W$. $(H$.) hertigi que reposaba en el Museo de Entomología de la "Florida State Collection of Arthropods (FSCA)" en Gainesville, Estados Unidos, integrado por un holotipo macho rotulado como " $W$. hertigi, holotype, male, \# 1228, Rio Chico Hydro Sta., Upper Rio Chagres, Panama, 20 March 48, in rock crevice near stream", un alotipo hembra rotulado como " $W$. hertigi, allotype, female, \# 3444, La Roca, Costa Rica, 29 April 51, buttresses, Rosabal colp', así como machos y hembras de ambos países.

\section{Resultados}

Se identificaron taxonómicamente cinco machos y dos hembras del subgénero Hertigia de Warileya, los cuales fueron determinados como $W$. (H.) hertigi. En ambos sexos fue notoria la ausencia de cicatrices de setas en el anepisternón, el proepimerón y el clípeo, la presencia de dos hileras transversales de cicatrices de setas en los tergitos, y la longitud corta $(5-25 \mu \mathrm{m}, \mathrm{n}=6)$ de la vena gamma, con la bifurcación de $R_{2+3+4}$ justo después de la inserción de la vena transversal r-m (figura 1A). Estos también presentaron sutura interocular completa, faringe con espinas pequeñas, y fórmula 


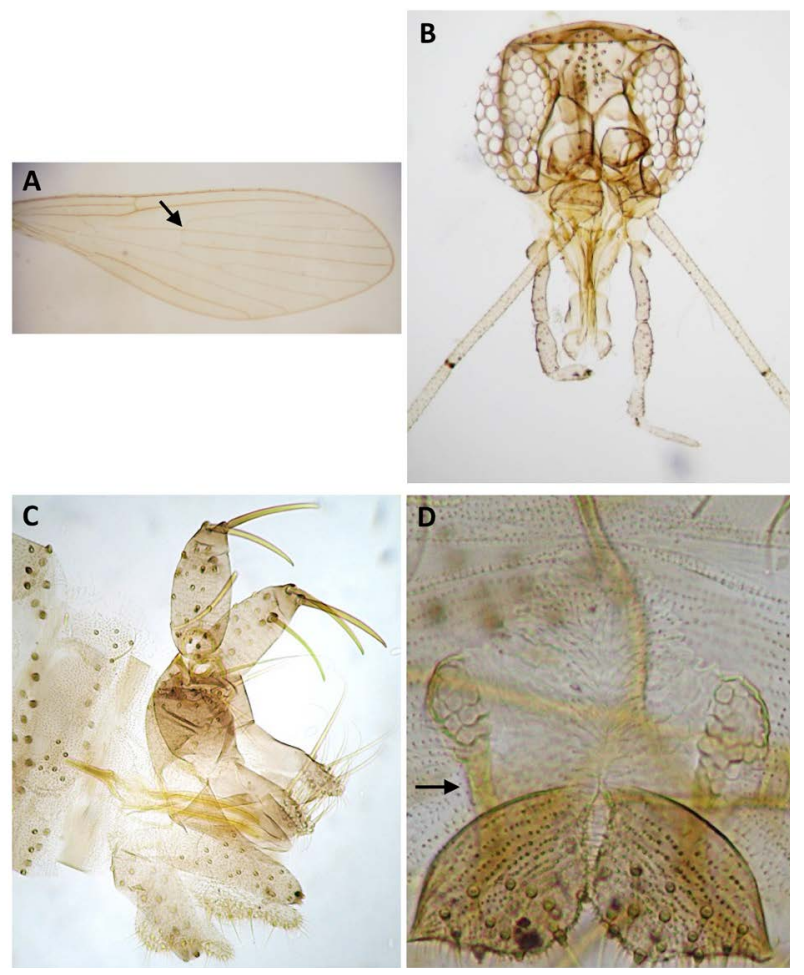

Figura 1. Adultos de Warileya (Hertigia) hertigi (Fairchild, 1949), recolectados en el departamento de Antioquia, Colombia. A: Ala de la hembra, en la cual se señala la vena gamma (flecha negra); B: Cabeza de la hembra; C: Terminalia del macho; D: Abdomen de la hembra, en el cual se muestra la gonapófisis del octavo esternito (flecha negra).

palpal 1.4.(2.3).5 en tres y 1.4.2.3.5 en dos, con el quinto palpómero (63-68 $\mu$ en tres machos; $75-76 \mu$ en dos hembras) ligeramente superior en longitud al tercero (55-65 $\mu$ en tres machos; $85 \mu$ en dos hembras) (figura $1 \mathrm{~B}$ ).

Los machos se caracterizaron por presentar la coxita (78-88 $\mu \mathrm{m}$, en tres) más corta que el estilo (99-103 $\mu \mathrm{m}$, en cuatro), este último provisto de dos espinas apicales y una preapical, y por tener el lóbulo lateral comparativamente más corto que el cerco, pero más largo que el parámero (figura 1C), que se observó recubierto de setas en su parte distal. Además, los machos exhibieron filamentos genitales cortos (94-115 $\mu$ en cinco), equivalentes a 1,2-1,7 veces la longitud del apodema eyaculador más la bomba genital (68-81 $\mu$, en cinco), los cuales estuvieron asociados a un par de varas esclerotizadas, dispuestas paralelas a estos y la bomba genital.

En la genitalia de las hembras, se destacó el brazo central de la furca genital con el ápice no dilatado, observado en posición ventral, y la presencia de un par de proyecciones internas esclerotizadas, paralelas a la furca genital, denominadas tradicionalmente como ganopófisis del octavo esternito $(9,17)$. Estas proyecciones esclerotizadas adoptan apicalmente el aspecto de mórula y convergen en una sola estructura membranosa y rugosa, en forma piramidal, que se prolonga hasta superar la mitad del brazo central de la furca genital (figura 1D).

\section{Discusión}

Los flebotomíneos del género Warileya se caracterizan por presentar alas anchas, con el ápice generalmente redondeado y la bifurcación $R_{2+3+4}$ cercana a la inserción de la vena transversal $r-m$, sutura interocular completa, pleura sin setas en el anepisternón y el proepimerón, tergitos con dos hileras transversales de setas, y genitales masculinos pequeños, con la coxita más corta que el estilo.

Lewis, et al. (18), reconocieron la existencia de dos subgéneros dentro de Warileya, el subgénero Warileya, que actualmente está compuesto por W. (W.) phlebotomanica Hertig, 1948, W. (W.) rotundipennis, $W$. (W.) nigrosacculus Fairchild y Hertig, 1951, W. (W.) yungasi Velasco y Trapido, 1974, W. (W.) fourgassiensis Le Pont y Desjeux, 1984, W. (W.) lumbrerasi Ogusuku, Pérez, Davies y Villaseca, 1996, W. (W.) euniceae Fernández, Carbajal, Astete y Wooster, 1998, y W. (W.) leponti Galati y Cáceres, 1999, y el subgénero Hertigia, integrado sólo por $W$. (H.) hertigi. Este género está representado en Colombia por tres especies, Warileya $(W$.$) nigrosacculus, W$. (W.) rotundipennis y W. (H.) hertigi (19).

Hertigia fue descrito inicialmente en la categoría taxonómica de género (8). Sin embargo, el posterior hallazgo de una especie de Warileya con características que habían sido propuestas como distintivas del género Hertigia (12), conllevó la degradación del último al estatus de subgénero (18). Posteriormente, Artemiev (20) retomó la clasificación original de este taxón y lo restituyó al rango de género, categorización que fue acogida por Galati (3). No obstante, en el presente trabajo se siguió el tratamiento tradicional de Hertigia como subgénero de Warileya, a la espera de estudios moleculares que permitieran esclarecer la relación entre estos.

El monotípico subgénero Hertigia es uno de los taxones de la subfamilia Phlebotominae menos frecuente en los muestreos entomológicos, más aún, los dos últimos registros de $W$. $(H$.) hertigi 
datan de principios de la década del noventa, en Costa Rica (21), y de finales de la década del 2000, en el municipio de San Antonio del Tequendama, departamento de Cundinamarca, Colombia (19). No obstante, desde el punto de vista taxonómico, esta representa una de las especies de mayor importancia, por cuanto reúne los caracteres morfológicos más ancestrales de la subfamilia $(8,20)$. Los ejemplares de $W$. $(H$.) hertigi recolectados en Antioquia, Colombia, exhibieron las características diagnósticas de la especie, referidas en la descripción original de ambos sexos $(8,10)$. En este sentido, es necesario precisar que, aunque el par de proyecciones esclerotizadas observadas al nivel del octavo esternito en las hembras no fueron incluidas en la ilustración original de la hembra de $W$. (H.) hertigi (10), sí están presentes en el alotipo examinado.

Respecto a la longitud de la vena gamma, con la bifurcación de $R_{2+3+} 4$ justo después de la inserción de la vena transversal $\mathrm{r}-\mathrm{m}$, en contraste con el holotipo de $W$. (H.) hertigi en el cual la bifurcación se produce antes de la vena transversal $r-m$, se debe indicar que esta variación había sido previamente registrada en el material tipo de la especie (10). También, es destacable el poliformismo en la fórmula palpal, que correspondió a 1.4.2.3.5 en los especímenes de Colombia y a 1.4.2.5.3 en los ejemplares tipo de la especie, con la longitud del palpómero quinto levemente mayor que la del tercero en los flebotomíneos de Colombia. Lo anterior se explica por el tamaño similar de estos segmentos palpales, por lo cual cambios menores en su longitud ocasionan modificaciones en la fórmula palpal. Cabe resaltar que en una especie congénere, $W$. (W.) lumbrerasi, se ha observado una gran desviación estándar en el tamaño del palpómero quinto (14).

En conclusión, se registra por primera vez la presencia de $W$. $(H$.$) hertigi en Antioquia, Colombia,$ lo cual incrementa a 64 el número de especies de flebotomíneos encontradas en este departamento (2). En total, 164 especies de flebotomíneos han sido halladas en Colombia.

\section{Conflicto de intereses}

No hay conflicto de intereses.

\section{Financiación}

Este trabajo fue financiado por el Programa de Estudio y Control de Enfermedades Tropicales (PECET) de la Universidad de Antioquia.

\section{Referencias}

1. Lainson R, Shaw JJ. New World Leishmaniasis. In: Cox FEG, Wakelin D, Gillespie SH, Despommier DD, editors. Topley \& Wilson's Microbiology and Microbial Infections. 10th edition. London: John Wiley \& Sons, Ltd.; 2005. p. 313-49.

2. Bejarano EE, Estrada LG. Family Psychodidae. Zootaxa. 2016;4122:187-238. https://doi.org/10.11646/zootaxa.4122. 1.20

3. Galati EA. Phlebotominae (Diptera, Psychodidae). Classificação, morfologia, terminologia e identificação de Adultos. Apostila de Bioecologia e Identificação de Phlebotominae. São Paulo: Universidade de São Paulo; 2015. p. 130.

4. Moreno M, Ferro C, Rosales-Chilama M, Rubiano L, Delgado M, Cossio A, et al. First report of Warileya rotundipennis (Psychodidae: Phlebotominae) naturally infected with Leishmania (Viannia) in a focus of cutaneous leishmaniasis in Colombia. Acta Trop. 2015;148:191-6. https://doi.org/10.1016/j.actatropica.2015.04.017

5. Vivero RJ, Muskus CE, Uribe SI, Bejarano EE, Torres C. Flebotomíneos (Diptera: Psychodidae) en la Reserva Natural del Cañón del Río Claro (Antioquia), Colombia. Actual Biol. 2010;32:165-71.

6. Young DG, Duncan MA. Guide to the identification and geographic distribution of Lutzomyia sandflies in Mexico, the West Indies, Central and South America (Diptera: Psychodidae). Mem Amer Ent Inst. 1994;54:1-881.

7. Hertig M. A new genus of bloodsucking Psychodids from Perú (Diptera, Psychodidae). Ann Entomol Soc Am. 1948; 41:8-16. https://doi.org/10.1093/aesa/41.1.8

8. Fairchild GB. A new fly related to Phlebotomus from Panamá (Diptera, Psychodidae). Proc Entomol Soc Washington. 1949;51:81-4.

9. Fairchild GB, Hertig M. Notes on the Phlebotomus of Panamá (Diptera, Psychodidae). VIII. Two new species of Warileya. Ann Entomol Soc Am. 1951;44:422-9. https://doi. org/10.1093/aesa/44.3.422

10. Fairchild GB. A note on Hertigia hertigi Fairchild and description of the female (Diptera, Psychodidae). Proc Entomol Soc Washington. 1953;55:101-2.

11. Young DG, Chaniotis BN. The male of Warileya nigrosacculus (Diptera: Psychodidae). Fla Entomol. 1972;55:97-9.

12. Velasco J, Trapido H. Two new phlebotomine sandflies from Bolivia, Lutzomyia boliviana, n. sp. and Warileya yungasi, n. sp. (Diptera: Psychodidae). J Med Entomol. 1974;11:433-6.

13. Le Pont F, Desjeux P. Warileya fourgassiensis n. $\mathrm{sp}$. (Diptera, Psychodidae), nouveau phlébotome cavernicole découvert en Guyane française. Cahiers ORSTOM. Sér Entomol Méd Parasitol. 1984;22:129-34.

14. Ogusuku E, Pérez JE, Davies CR, Villaseca P. Description of Warileya lumbrerasi $n$. sp. (Diptera: Psychodidae) from Perú. Mem Inst Oswaldo Cruz. 1996;91:711-6. http://dx.doi. org/10.1590/S0074-02761996000600010

15. Fernández R, Carbajal F, Astete H, Wooster MT. Notes on the phlebotomine sandflies from the Peruvian Southeast. II. 
Description of Lutzomyia (Helcocyrtomyia) wattsi sp. $\mathrm{n}$. and Warileya euniceae sp. n. (Diptera, Psychodidae).Rev Bras Entomol. 1998;42:65-70. http://dx.doi.org/10.1590/S007402761998000100009

16. Cáceres AG, Galati EA. Descrição de três espécies novas de Phlebotominae (Diptera, Psychodidae) do Departamento de Pasco, Perú. Rev Bras Entomol. 1999;43:293-9.

17. Sinton JA. Notes on some Indian species of the genus Phlebotomus. Part XIV. The hypopygium of the female Phlebotomus. Indian J Med Res. 1925:13;97-107.

18. Lewis DJ, Young DG, Fairchild GB, Minter DM. Proposals for a stable classification of the phlebotomine sandflies (Diptera: Psychodidae). Syst Entomol. 1977;2:319-32. http://dx.doi.org/10.1111/j.1365-3113.1977.tb00381.x
19. Delgado LM. Composición y abundancia de especies de Lutzomyia (Diptera: Psychodidae) en San Antonio del Tequendama, departamento de Cundinamarca, región Andina, cuenca del rio Magdalena (tesis). Tunja: Universidad Pedagógica y Tecnológica de Colombia; 2008. p. 69.

20. Artemiev MM. A classification of the subfamily Phlebotominae. Parassitologia. 1991;33:69-77.

21. Herrero MV, Rojas JC, Jiménez AE, Zeledón R, Pereira R, Gutiérrez H. II. Phlebotominae sandflies (Diptera: Psychodidae: Phlebotominae) associated with human houses in an endemic area for cutaneous leishmaniasis in Costa Rica. En: Wijeyaratne P, Godman T, Espinal C, editores. Leishmaniasis control strategies: A critical evaluation of IDRC-supported research. Ottawa: International Development Research Center; 1992. p. 229-41. 